\title{
Machine Learning Applications in Endocrinology and Metabolism Research: An Overview
}

\author{
Namki Hong, Heajeong Park, Yumie Rhee
}

Division of Endocrinology and Metabolism, Department of Internal Medicine, Yonsei University College of Medicine, Seoul, Korea

Machine learning (ML) applications have received extensive attention in endocrinology research during the last decade. This review summarizes the basic concepts of ML and certain research topics in endocrinology and metabolism where ML principles have been actively deployed. Relevant studies are discussed to provide an overview of the methodology, main findings, and limitations of ML, with the goal of stimulating insights into future research directions. Clear, testable study hypotheses stem from unmet clinical needs, and the management of data quality (beyond a focus on quantity alone), open collaboration between clinical experts and ML engineers, the development of interpretable high-performance ML models beyond the black-box nature of some algorithms, and a creative environment are the core prerequisites for the foreseeable changes expected to be brought about by ML and artificial intelligence in the field of endocrinology and metabolism, with actual improvements in clinical practice beyond hype. Of note, endocrinologists will continue to play a central role in these developments as domain experts who can properly generate, refine, analyze, and interpret data with a combination of clinical expertise and scientific rigor.

Keywords: Machine learning; Artificial intelligence; Deep learning; Endocrinology; Metabolism; Diabetes; Osteoporosis; Pituitary; Adrenal; Thyroid

\section{INTRODUCTION}

The use of machine learning (ML) applications in various fields of health research are rapidly expanding, and ML has the potential to improve the current health system and clinical practice. In endocrinology and metabolism research, the number of publications on ML has exponentially increased, reaching roughly 2,000 publications by the end of the last decade (PubMed query: Search ((((((“Machine Learning”[Mesh]) OR “Artificial Intelligence” [Mesh]) OR “Deep Learning”[Mesh])) OR (((machine learning [Title/Abstract]) OR artificial intelligence[Title/Abstract]) OR deep learning[Title/Abstract]))) AND ((()((((endocrinology[Title/

Received: 6 February 2020, Revised: 15 February 2020,

Accepted: 21 February 2020

Corresponding author: Namki Hong

Department of Internal Medicine, Yonsei University College of Medicine,

50-1 Yonsei-ro, Seodaemun-gu, Seoul 03722, Korea

Tel: +82-2-2228-0790, Fax: +82-2-393-6884, E-mail: nkhong84@yuhs.ac
Abstract]) OR diabetes[Title/Abstract]) OR pituitary[Title/Abstract]) OR thyroid[Title/Abstract]) OR adrenal gland[Title/Abstract]) OR osteoporosis[Title/Abstract])) OR (((()(“Endocrinology" [Mesh]) OR "Diabetes Mellitus"[Mesh]) OR "Pituitary Gland" [Mesh]) OR "Thyroid Gland”[Mesh]) OR “Adrenal Glands" [Mesh]) OR “Osteoporosis”[Mesh])); search date: January 1st, 1986 to January 17th) (Fig. 1). The accumulation of structured or unstructured medical data, the exponential growth of available computing power, and the availability of useful open resources for implementing ML have contributed to the expanding ML applications in health care. In this review, a brief overview of the basic concepts of ML, exemplary studies of ML applications in

Copyright $\odot 2020$ Korean Endocrine Society

This is an Open Access article distributed under the terms of the Creative Commons Attribution Non-Commercial License (https://creativecommons.org/ licenses/by-nc/4.0/) which permits unrestricted non-commercial use, distribution, and reproduction in any medium, provided the original work is properly cited. 


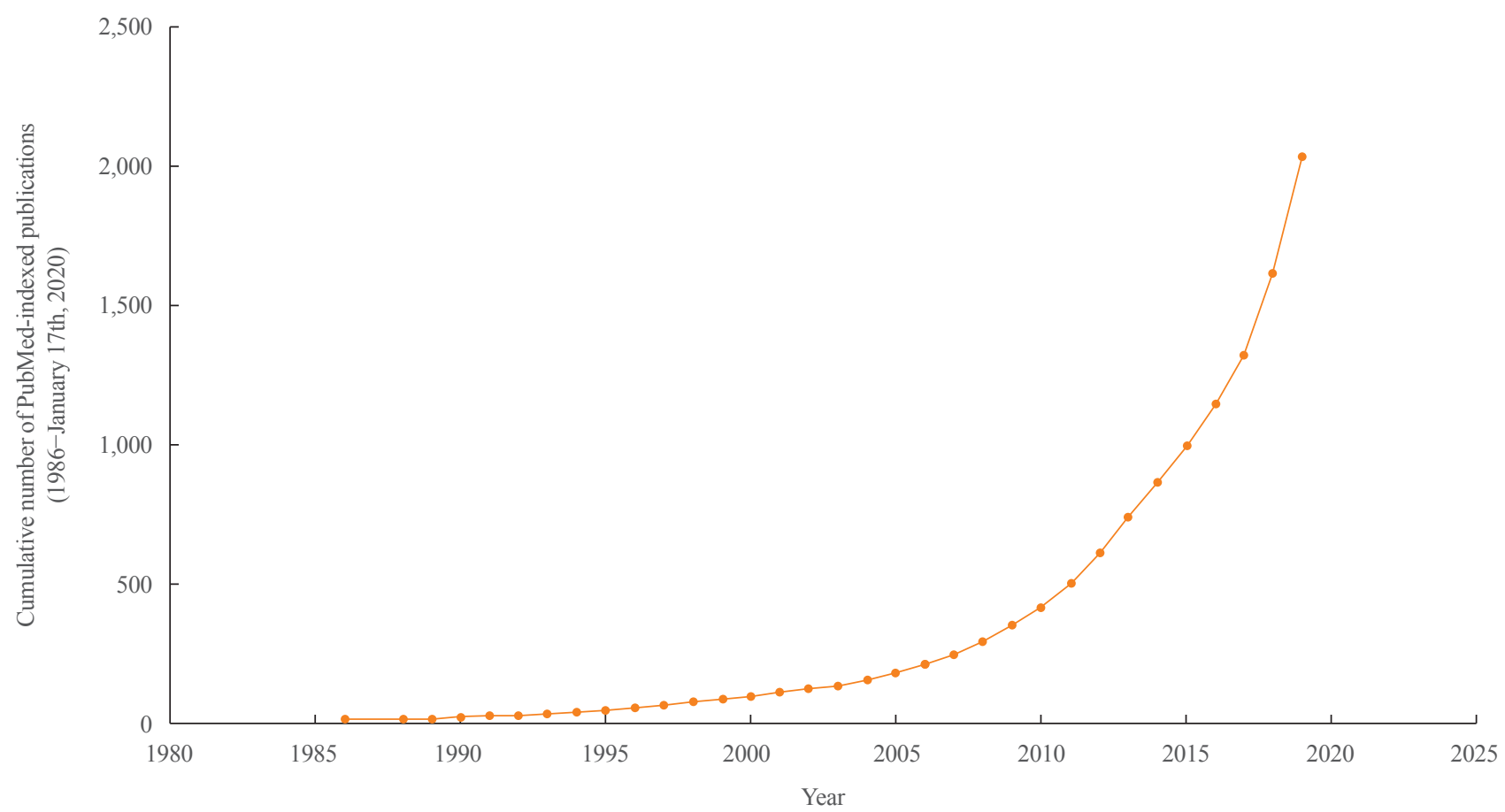

Fig. 1. The increasing trend in the number of artificial intelligence or machine learning-related publications per year in the endocrinology and metabolism field. The included publications were confined to PubMed-indexed records until the search date (January 17th, 2020), with combinations of search terms including machine learning, artificial intelligence, deep learning, endocrinology, metabolism, diabetes, pituitary, thyroid, adrenal gland, and osteoporosis, using PubMed query as follows: search ((()((“Machine Learning”[Mesh]) OR “Artificial Intelligence”[Mesh]) OR “Deep Learning”[Mesh])) OR (((machine learning[Title/Abstract]) OR artificial intelligence[Title/Abstract]) OR deep learning[Title/Abstract]))) AND ((((((((endocrinology[Title/Abstract]) OR diabetes[Title/Abstract]) OR pituitary[Title/Abstract]) OR thyroid[Title/Abstract]) OR adrenal gland[Title/Abstract]) OR osteoporosis[Title/Abstract])) OR ((((((“Endocrinology”[Mesh]) OR “Diabetes Mellitus”[Mesh]) OR “Pituitary Gland”[Mesh]) OR “Thyroid Gland”[Mesh]) OR “Adrenal Glands”[Mesh]) OR “Osteoporosis”[Mesh])).

endocrinology research, and related perspectives will be provided for endocrinologists and clinical practitioners who are becoming interested in the principles of ML.

\section{MACHINE LEARNING: A BRIEF INTRODUCTION}

Artificial intelligence, machine learning, and deep learning The terms "artificial intelligence (AI)," "ML," and "deep learning" are often used concomitantly and sometimes interchangeably in the medical literature. The U.S. Food and Drug Administration defined $\mathrm{AI}$ as "the science and engineering of making intelligent machines, especially intelligent computer programs," based on the definition proposed by McCarthy [1,2]. Learning and reasoning are the main functions that intelligence refers to in this context, although intelligence more broadly includes selfawareness, introspection, action, heuristics, and practical knowledge [1]. ML is defined as an "AI technique that can be used to design and train software algorithms to learn from and act on data," as a subset of AI. Therefore, all ML counts as AI, but not all AI involves ML. Deep learning, also known as deep neural networks, refers to a subset of ML algorithms implemented by stacked multilayer neural networks, mimicking the neural architecture of the human brain. As noted in a summary report from the Third Annual Machine Learning for Health Workshop held in December 2018, traditional technical researchers and communities appear to favor the term "ML" when describing the methodology underlying their work. However, clinicians tend to prefer using "AI" as an umbrella term in the medical literature, and this discrepancy might need to be resolved in order to remove potential terminological barriers and to prevent unnecessary confusion among research communities [3].

\section{Machine learning algorithms and performance metrics}

ML algorithms can be classified into four main categories: supervised, semi-supervised, unsupervised, and reinforcement learning (Table 1) [4-7]. Supervised learning requires a labeled dataset with output mapped to input to train a function. The goal 


\begin{tabular}{|c|c|c|c|c|}
\hline Types of learning & Supervised learning & Semi-supervised learning & Reinforcement learning & Unsupervised learning \\
\hline Concept & $\begin{array}{l}\text { Learning a function that best } \\
\text { approximates new input to } \\
\text { the desired output based on } \\
\text { a given relationship } \\
\text { between the input and } \\
\text { labeled output from the } \\
\text { labeled dataset }\end{array}$ & $\begin{array}{l}\text { A mixed approach of super- } \\
\text { vised and unsupervised } \\
\text { learning applicable to a } \\
\text { small amount of labeled data } \\
\text { and a large amount of unla- } \\
\text { beled data }\end{array}$ & $\begin{array}{l}\text { Learning by maximizing the } \\
\text { reward function based on } \\
\text { the responses yielded by } \\
\text { various actions to achieve } \\
\text { arbitrary goals in a given } \\
\text { unstructured or unknown } \\
\text { environment }\end{array}$ & $\begin{array}{l}\text { Finding structures or patterns in an } \\
\text { unlabeled dataset }\end{array}$ \\
\hline Common tasks & Regression, classification & Regression, classification & $\begin{array}{l}\text { Taking actions to maximize } \\
\text { the reward }\end{array}$ & $\begin{array}{l}\text { Clustering, dimensionality } \\
\text { reduction }\end{array}$ \\
\hline Estimators & $\begin{array}{l}\text { Naive Bayesian, k-nearest } \\
\text { neighbors, decision tree, } \\
\text { support vector machine } \\
\text { (SVM), neural network, } \\
\text { logistic/ridge/linear } \\
\text { regression, elastic net, etc. }\end{array}$ & $\begin{array}{l}\text { Generative model, semi-su- } \\
\text { pervised SVM, etc. }\end{array}$ & $\begin{array}{l}\text { Q-learning, policy gradient, } \\
\text { actor-critic, etc. }\end{array}$ & $\begin{array}{l}\text { K-means, density-based spatial } \\
\text { clustering of applications with } \\
\text { noise (DBSCAN), auto-encod- } \\
\text { ers, deep Boltzmann machine, } \\
\text { principal component analysis, } \\
\text { locally linear embedding, etc. }\end{array}$ \\
\hline Examples & $\begin{array}{l}\text { Prediction of gestational } \\
\text { diabetes according to } \\
\text { biochemical test results } \\
\text { based on simple features } \\
\text { extracted from an electronic } \\
\text { health records database [4] }\end{array}$ & $\begin{array}{l}\text { The DeepHeart algorithm [7], } \\
\text { which provides cardiovas- } \\
\text { cular risk scores based on } \\
\text { heart rate monitoring from } \\
\text { popular wearable devices } \\
\text { (Fitbit, Apple Watch, etc.) }\end{array}$ & $\begin{array}{l}\text { Determining the optimal } \\
\text { insulin dose in patients } \\
\text { with type } 1 \text { diabetes based } \\
\text { on activity, hemoglobin } \\
\text { Alc level, alcohol con- } \\
\text { sumption status, and the } \\
\text { previous insulin dose [5] }\end{array}$ & $\begin{array}{l}\text { Identifying novel clusters or } \\
\text { biomarkers based on various } \\
\text { features collected by an unbiased } \\
\text { multimodal approach, which } \\
\text { finds differences in risks for } \\
\text { certain diseases compared to } \\
\text { other groups [6] }\end{array}$ \\
\hline
\end{tabular}

of supervised learning is to derive a function that infers the most desired output for new input from the previously labeled dataset. Unsupervised learning explores structures or patterns in unlabeled datasets to achieve clustering or dimensionality reduction. Semi-supervised learning is a blend of those two approaches that is suitable for datasets with a small amount of labeled data and extensive unlabeled data. Reinforcement learning is suitable for finding optimal actions in an unstructured and complex environment by maximizing the cumulative rewards from actions taken in that environment. Unlike supervised learning, which is based on prior knowledge of input-output mapping at the start, a reinforcement learning function evolves sequentially by collecting information on every action-response relationship during the task. Although some guidance on selecting an estimator can be obtained from a so-called "cheat-sheet" for initial ML estimators (scikit-learn; https://scikit-learn.org/stable/tutorial/machine_ learning_map/index.html), a one-size-fits-all approach may not be applicable in most cases. Estimators are often chosen through an iterative process that takes into account the quantity, structure, and extendibility of the dataset, the characteristics of the research hypothesis or problems, the performance of trained functions, and researchers' experiences and intuition (Fig. 2). Choosing the performance metrics that best suit the research purpose is another task that needs to be carefully accomplished after establishing an ML model (Supplemental Table S1) [8-11].

\section{MACHINE LEARNING APPLICATIONS IN ENDOCRINOLOGY AND METABOLISM}

Results of the text analysis on the titles of literature were presented as Fig. 3. The titles of 611 studies (English language, human study, not a review or meta-analysis) published within the last 5 years were parsed to count the frequency of words that appeared. Among a total of 2,115 words, the top 30 words with high frequency were analyzed. Among diseases, 'diabetes' or 'diabetic' appeared most frequently among the top 30 words (52\%), followed by retinopathy (14\%), thyroid (14\%), carcinoma (8\%), and osteoporosis (7\%). Regarding ML tasks, 'risk prediction' or 'predict' accounted for $31 \%$, and the composite of 'detection,' 'classification,' 'identification,' and 'diagnosis' reached up to $40 \%$, followed by 'segmentation' (5\%) or 'bioinformatics' $(7 \%)$. In this section, studies were summarized to present some exemplary cases in utilizing ML applications in the endocrinology and metabolism field. Seventeen studies were arbitrarily chosen on the basis of (1) the balance between disease fields (diabetes, thyroid, pituitary, and bone and mineral disor- 


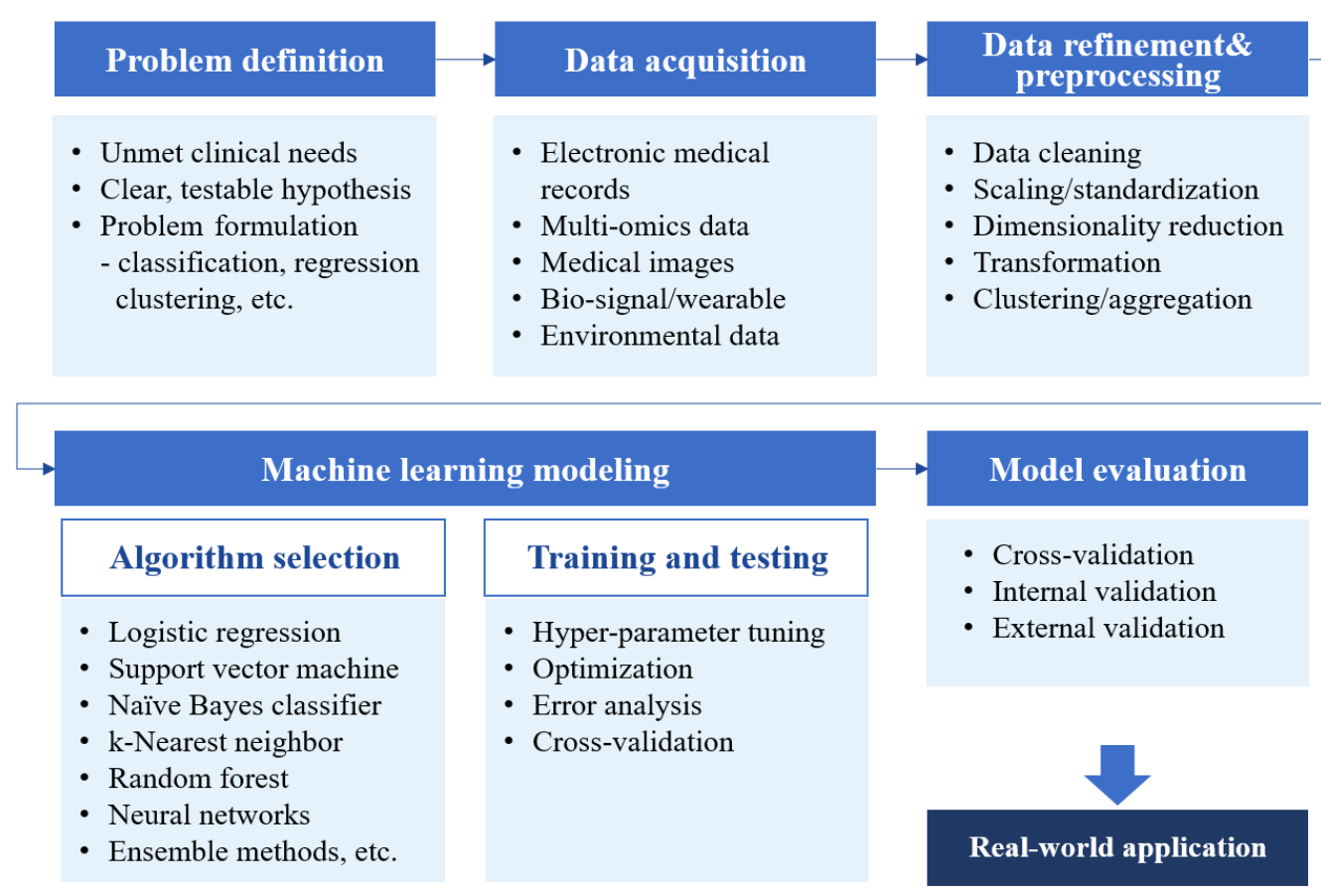

Fig. 2. A brief workflow of machine learning-based medical research.

ders), (2) inclusion of at least one study which illustrates the various types of ML applications (supervised, unsupervised, and reinforcement learning), and (3) the publication date within last 3 years. The research topics were categorized into screening and diagnosis, risk prediction, and translational research based on frequently appeared ML tasks from text analysis (Fig. 3), with subcategories that were not mutually exclusive in some cases. It should be noted that the selected studies might not be sufficient to reflect an entire trend of ML applications in the endocrinology field, but it can provide practical examples for understanding the utility of ML algorithms applied to various fields of endocrine researches. The details of the reviewed studies are summarized in Table 2 [4-6,12-26].

\section{Screening and diagnosis}

\section{Improvement of screening strategies}

The development of efficient screening tools for endocrine disorders may have clinical impacts, both in terms of improved prognoses of individual patients through disease detection at an earlier stage and the cost-effective allocation of public health resources by focusing on individuals with a high risk of disease and avoiding unnecessary testing in low-risk groups. Researchers have sought to determine whether ML algorithms are able to provide a better way of screening for various endocrine diseases. Artzi et al. [4] provided an excellent example of applying the principles of ML to find useful screening tools for gestational diabetes based on a sizeable electronic health record (EHR) database. EHR data of 588,622 pregnancies from 368,351 women collected at the nationwide level in Israel between 2010 and 2017 were used to train an ML model to predict the risk of gestational diabetes. Among 2,355 candidate features, the researchers developed a simple model consisting of only nine self-reportable questions (without previous laboratory results in some cases) based on a gradient boosting model, which showed fair discriminatory performance (area under the receiver operating characteristic curve, 0.80 vs. 0.68 for the conventional glucose challenge test at 24 to 28 weeks of gestation) even at an earlier time point relative to the initiation of pregnancy. Medical image data have the potential to provide features suitable for the opportunistic screening of endocrine disorders. Valentinitsch et al. [12] trained an ML model to identify individuals with prevalent vertebral fractures based on non-fractured vertebral regions in computed tomography scans taken for various purposes. By combining global and local density and texture parameters, the ML model outperformed volumetric bone mineral density (BMD) alone in discriminating the presence of vertebral fractures, suggesting the potential of a semi-automated pipeline for the opportunistic screening of individuals with a high risk of fracture. Kong et al. [13] developed an ML model to detect facial features from photos of patients with acromegaly, and their 


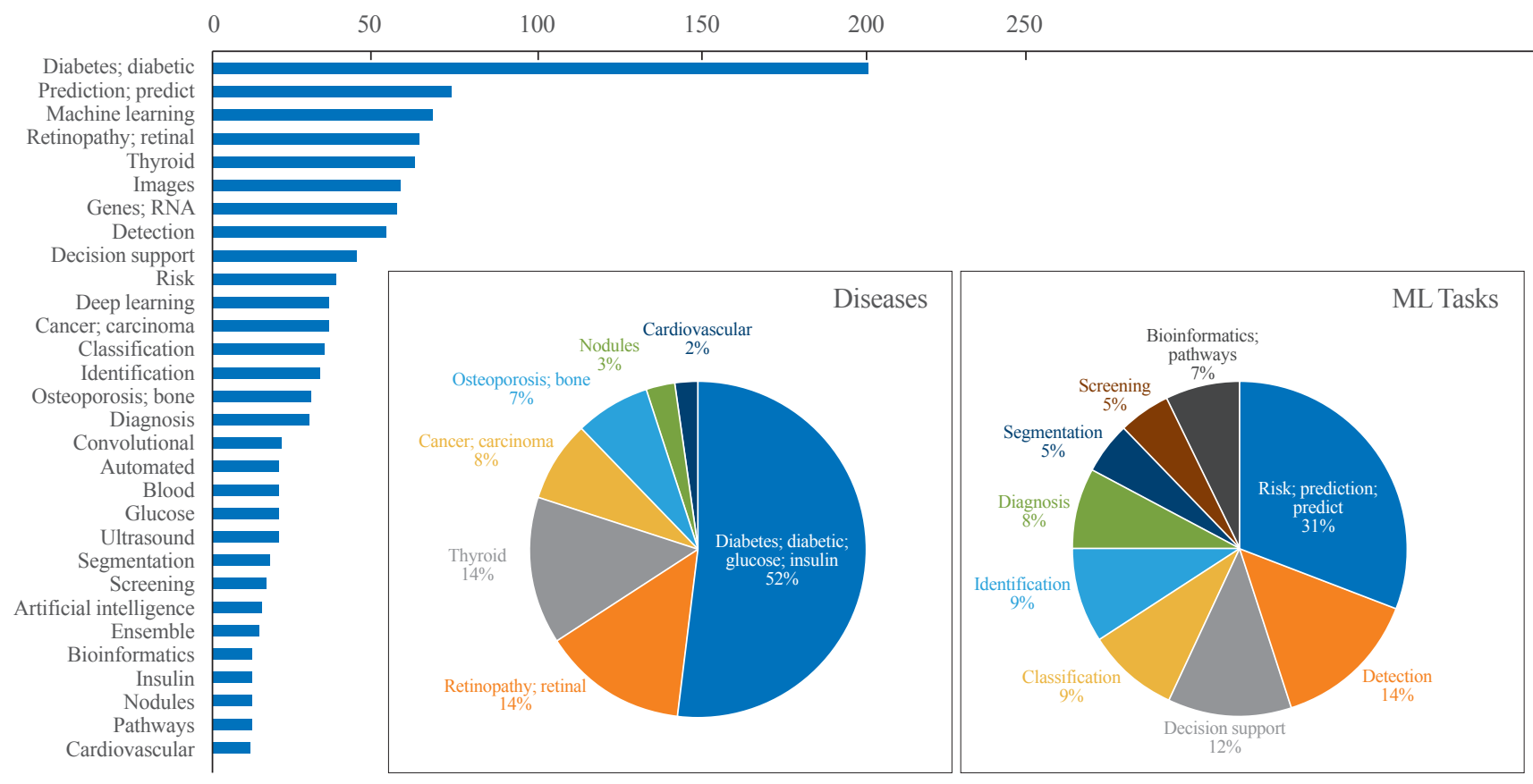

Fig. 3. Top 30 frequently appeared words in the titles of machine learning (ML)-based endocrinology studies between 2015 and 2019. Among a total of 2028 literatures searched by PubMed query a on Jan 17th, 2020, text analysis was performed with nouns and adjectives parsed from the titles of 611 studies (English language, human study without review or meta-analysis) published within last 5 years. Cumulative counts of appearance of top 30 words were plotted as horizontal bar plot. Frequently appeared diseases and ML tasks were plotted as pie charts separately. 'PubMed query: (Search ((()((“Machine Learning”[Mesh]) OR “Artificial Intelligence”[Mesh]) OR “Deep Learning”[Mesh])) OR (((machine learning[Title/Abstract]) OR artificial intelligence[Title/Abstract]) OR deep learning[Title/Abstract]))) AND (((()((endocrinology[Title/Abstract]) OR diabetes[Title/Abstract]) OR pituitary[Title/Abstract]) OR thyroid[Title/Abstract]) OR adrenal gland[Title/Abstract]) OR osteoporosis[Title/Abstract])) OR ((((((“Endocrinology”[Mesh]) OR “Diabetes Mellitus”[Mesh]) OR “Pituitary Gland”[Mesh]) OR “Thyroid Gland”[Mesh]) OR “Adrenal Glands”[Mesh]) OR “Osteoporosis”[Mesh])).

model may have the potential to help the detection of acromegaly at an earlier stage.

\section{Facilitating the diagnostic workflow}

Tackling the gray area of diagnostic uncertainty with new modalities has always been an important task for clinicians. Asymptomatic hyperparathyroidism can be challenging to identify without a high index of suspicion because it involves subtle biochemical changes and its phenotype overlaps with those of primary osteoporosis and other rare mineral disorders, including familial hypocalciuric hypercalcemia [27]. Somnay et al. [25] trained an ML model to identify patients with primary hyperparathyroidism among patients who underwent neck surgery, including thyroidectomy or parathyroidectomy, although relatively low performance was shown for mild disease. Several studies have shown that ML could support the decision process of whether to perform an invasive biopsy on a thyroid nodule based on ultrasonography, with good classification performance similar to that of radiology experts; therefore, ML classifications might potentially provide guidance to operators during data acquisition and measurement $[14,28]$. A well-validated, accurate, non-invasive ML model may have the potential to replace standard invasive diagnostic modalities for certain diseases. For instance, the global burden of nonalcoholic fatty liver disease (NAFLD) is rapidly growing, but invasive liver biopsy remains the gold standard for diagnosing NAFLD and nonalcoholic steatohepatitis. Perakakis et al. [15] developed a support vector machine-based model to classify NAFLD based on features obtained from the lipidomic, glycomic, and liver fatty acid analysis of serum samples. For the presence of liver fibrosis, a parsimonious exploratory model with 10 lipid species showed high accuracy (up to 98\%), suggesting the possibility of a targeted lipidomic approach as an alternative non-invasive diagnostic tool, although the model needs to be further validated in other ethnicities and individuals with a milder spectrum of liver diseases [29]. 
Table 2. Summary of Recent Studies Related to Machine Learning Applications in the Endocrinology Field

\begin{tabular}{|c|c|c|}
\hline Task & Study (disease field) & Study subjects \\
\hline $\begin{array}{l}\text { Screening and } \\
\text { diagnosis }\end{array}$ & $\begin{array}{l}\text { Artzi et al. (2020) } \\
\text { [4] (Diabetes and } \\
\text { related disorders) }\end{array}$ & $\begin{array}{l}\text {-Retrospective nationwide } \\
\text { electronic health record } \\
\text { data of } 588,622 \text { pregnancies } \\
\text { from } 368,351 \text { women be- } \\
\text { tween } 2010 \text { to } 2017 \text { in Isra- } \\
\text { el including data of demo- } \\
\text { graphics, anthropometrics, } \\
\text { laboratory tests, diagnoses, } \\
\text { and pharmaceuticals } \\
\text {-Internal validation set } \\
\text { ( } n=137,220 \text {; with geo-tem- } \\
\text { poral difference) }\end{array}$ \\
\hline
\end{tabular}

Design and method

-Aim: to establish an ML model Key implications

to improve the prediction of

gestational diabetes based on

electronic health record vs. a

conventional screening tool

-Reference labels: gestational

diabetes diagnosis by a two-step

approach (glucose challenge

test and oral glucose tolerance

test at 24-28 weeks of gesta-

tion)

-Comparator: National Institute of Health seven-item questionnaire

-Methods: supervised learning; gradient boosting model

De Silva et al.

(2020) [24]

(Diabetes and

-National Health and Nutrition Examination Survey

related disorders) (NHANES) 2013-2014

$(n=6,346)$

-Internal validation set

$(n=3,172)$

-External validation set:

NHANES 2011-2012

$(n=3,000)$
-Aim: to identify predictors of prediabetes to build a screening model

-Reference label: prediabetes defined using fasting plasma glucose, an oral glucose tolerance test, or hemoglobin A1c (HbAlc) according to American Diabetes Association recommendations

-Comparator: national prediabetes screening instrument -Methods: supervised learning; logistic regression, artificial neural network, random forests, gradient boosting
-ML was useful in developing a simple ninequestion model in self-reportable format from the large electronic health record dataset, which outperformed the current standard screening tool (AUROC 0.80 vs. 0.68).

-May facilitate early-stage interventions for women at high risk for gestational diabetes -May aid construction of a selective, costeffective screening approach according to predicted gestational diabetes risk instead of the current universal screening approach Limitations

-Inherent bias from retrospective electronic health record data review

-Performance might be different when based on actual self-reported surveys.

Key implications

-ML-based models had modest performance in discriminating inidividuals with prediabetes, which were comparable to current screening instrument (AUROC 0.70 vs 0.64 ). -An application of feature selection methods and machine learning algorithms to open dataset

-Novel predictors of prediabetes such as serum calcium, hysterectomy, hepatitis B were suggested by the feature selection algorithm; may provide new insights, but need to be cautious about unobserved confounding. Limitations

-Generalizability to other countries cannot be guaranteed.

-A more parsimonious model would be useful as a screening tool.

Key implications

-ML model with global and local density and texture parameters showed better performance in identifying individuals with vertebral fractures compared to using volumetric BMD alone (AUROC 0.88 vs 0.64).

-Proposed a quantitative, automatic pipeline for opportunistic CT screening for individuals with vertebral fractures

Limitations

-Consisted of oncologic patients; whether the pipeline is applicable to the general population needs to be validated.

-DXA data were not available; comparison with DXA and FRAX was not possible. 
Table 2. Continued

\begin{tabular}{llll}
\hline Task & Study (disease field) & \multicolumn{1}{c}{ Study subjects } & \multicolumn{1}{c}{ Design and method } \\
\hline Somnay et al. & -Retrospective cohort of & -Aim: to establish an ML model \\
(2017) [25] (Bone & patients $(n=6,777)$ with & discriminating patients with \\
and mineral & confirmed primary hyper- & primary hyperparathyroidism \\
disorders) & parathyroidism who under- & among patients who underwent \\
& went parathyroidectomy vs. & neck surgery \\
& controls $(n=5,033)$ who & - Reference label: surgically \\
& underwent thyroidectomy & confirmed primary hyperpara- \\
& from March 2001 to August & thyroidism \\
& 2013 & -Comparator: not applicable \\
& -Internal validation with 10- & -Methods: supervised learning; \\
& fold cross-validation & naive Bayesian network with \\
& & adaptive boosting
\end{tabular}

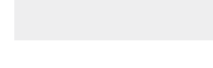

Buda et al. (2019)

[14] (Thyroid diseases)

Kong et al. (2018)

[13] (Pituitary diseases)

\section{-Retrospective cohort of}

1,377 thyroid nodules from 1,230 patients with complete imaging and conclusive cytologic or histologic diagnoses from August 2006 to May 2010 (training set: 1,278 nodules in 1,139 patients between 2006 and 2009)

-Internal validation set: 99 nodules in 91 consecutive patients (year 2009-2010)
-Aim: to provide biopsy recommendations for thyroid nodules based on two orthogonal ultrasound images

-Reference label: cytologically or histologically confirmed malignant or benign nodules on fine-needle aspiration (or surgical specimen where available) -Comparator: decisions from three Thyroid Imaging Reporting and Data System committee experts; nine individual radiologists in clinical practice

-Methods: supervised learning; region-based convolutional neural network, multi-task convolutional neural network

-Aim: to detect acromegaly from facial photographs -Reference label: biochemically proven acromegaly by growth hormone suppression testing with IGF-1 levels -Comparator: nine board-certified endocrinologists or neurosurgeons specializing in pituitary disease only through a photograph

-Methods: supervised learning; an ensemble of outputs from logistic regression, k-nearest neighbor, support vector machine, random forest, and convolutional neural network
Key finding and limitation

Key implication

-ML model helped identifying individuals with primary hyperparathyroidism those who underwent neck surgery (accuracy 95.2\%; $71.1 \%$ in mild case).

-Tested algorithm performance in the context of various relevant clinical situations

Limitation

-Cases comprised only patients referred for parathyroidectomy; potential for selection bias cannot be excluded.

-Did not include cases of urinary calcium excretion or familial hypocalciuric hypercalcemia as controls.

Key implication

-The ML model yielded similar sensitivity $(87 \%$ vs. $87 \%)$ and specificity ( $52 \%$ vs. $51 \%$ ) to that of expert radiologists (AUROC 0.87 vs. 0.82).

-Showed potential that ML model may be helpful to support clinical decision to go on invasive procedure for thyroid nodule.

Limitation

-The final test set included a relatively small number of nodules, leading to wide confidence intervals.

-An external validation set for generalization was not available.

-Applicability of model during the testing in clinical practice need to be investigated.

Key implication

-The ML model showed better performance in discriminating acromegaly, from the earlier stage, based on only by facial photograph compared to pituitary disease specialists (F1score 0.96 vs. 0.87 ).

-May have the potential to facilitate early detection of acromegaly based on facial recognition.

Limitations

-Did not include side view images.

-Relatively small sample size as an imagebased study compared to 128,175 retinal images in the previous work [26]. -Model based on a single ethnicity; cannot be extrapolated to another ethnicity. 
Table 2. Continued

\begin{tabular}{|c|c|c|c|}
\hline Task & Study (disease field) & Study subjects & Design and method \\
\hline & $\begin{array}{l}\text { Perakakis et al. } \\
\text { (2019) [15] } \\
\text { (Diabetes and } \\
\text { related disorders) }\end{array}$ & $\begin{array}{l}\text {-Serum samples of } 49 \\
\text { healthy subjects and } 31 \\
\text { patients with biopsy- } \\
\text { proven NAFLD } \\
\text {-Internal validation with } \\
\text { three-fold cross-validation }\end{array}$ & $\begin{array}{l}\text {-Aim: to train models for the } \\
\text { non-invasive diagnosis of } \\
\text { NASH and liver fibrosis based } \\
\text { on circulating lipids, glycans, } \\
\text { fatty acids identified by LC- } \\
\text { MS/MS and biochemical } \\
\text { parameters } \\
\text {-Reference label: biopsy-proven } \\
\text { NAFLD } \\
\text {-Comparator: not applicable } \\
\text {-Methods: supervised learning; } \\
\text { one-vs-rest nonlinear support } \\
\text { vector machine models with } \\
\text { recursive feature elimination }\end{array}$ \\
\hline
\end{tabular}

Kruse et al. (2017) -Retrospective data from [16] (Bone and 10,775 subjects from the mineral disorders) national Danish patient database with information on DXA scans, medication reimbursements, healthcare use, and comorbidities of female subjects
-Aim: to detect patient clusters with a high risk of fracture using an unsupervised clustering algorithm based on DXA scans, medication, and health care claims dataset

-Reference label: not applicable -Comparator: not applicable -Methods: unsupervised learning; Ward's-based hierarchical agglomerative clustering
Key implications

-The ML model including 20 features consisted of lipidomics, glycans, and adiponectin yielded high accuracy up to $90 \%$ in discriminating healthy individuals from patients with NAFLD and NASH.

-May provide a low-risk cost-effective, noninvasive alternative method to liver biopsy. Limitations

-Validation cohort was not available.

-Needs to be further validated in a different population.

Key implications

-Unsupervised clustering identified four high risk clusters and two low-risk clusters among nine clusters, which had different patterns of medication usage, compliance, and clinical outcomes despite similar DXA results.

-May provide novel insights into establishing indications for DXA screening.

Limitations

-Potential temporal changes in pharmacological treatment pattern during the 15 -year observation period

-Inherent limitations of the secondary use of a claims dataset; could not ascertain actual consumption of medication by individual subjects.

Risk prediction Segar et al. (2019) [17] (Diabetes and related 8,756 Patients without hear failure at baseline from the ACCORD trial dataset disorders) (50\% training set; $50 \%$ internal validation set; conducted between 1999 to 2009)

-External validation set: 10,819 participants without prevalent heart failure from the ALLHAT trial
-Aim: to develop an ML model to predict incident heart failure among patients with type 2 diabetes

-Reference label: incident hospitalization or death due to heart failure (captured and adjudicated by two independent reviewer physicians during the trial)

-Comparator: not applicable -Methods: supervised learning; random survival forest-based model
Key implications

-The ML-based models showed modest performance in prediction for incident heart failure among patients with type 2 diabetes in the external validation set (C-index 0.70 to $0.74)$.

-Each 1-unit increment in the WATCH-DM score was associated with a $24 \%$ higher relative risk of heart failure within 5 years.

-Strength of analyzing a large number of participants from a well-phenotyped clinical trial population

Limitations

-Discrimination for heart failure with preserved ejection fraction was relatively low in the subgroup analysis.

-Temporal changes of heart failure biomarkers and medications could not be reflected in the model.

-Need to validate the model in lower-risk cohorts of individuals with type 2 diabetes. 
Table 2. Continued

\begin{tabular}{|c|c|c|c|c|}
\hline Task & Study (disease field) & Study subjects & Design and method & Key finding and limitation \\
\hline & $\begin{array}{l}\text { Su et al. (2019) [18] } \\
\text { (Bone and } \\
\text { mineral disorders) }\end{array}$ & $\begin{array}{l}\text {-5,977 Community-dwelling } \\
\text { American men aged } 65 \text { or } \\
\text { older (MrOS cohort) with } \\
\text { 10-year follow-up data } \\
\text {-Internal validation with 10- } \\
\text { fold cross-validation }\end{array}$ & $\begin{array}{l}\text {-Aim: to develop a risk classifi- } \\
\text { cation model for hip fracture } \\
\text { prediction in community-dwell- } \\
\text { ing men } \\
\text {-Reference label: incident hip } \\
\text { fracture validated by a central- } \\
\text { ized physician using radiology } \\
\text { reports or X-rays } \\
\text {-Comparator: FRAX hip fracture } \\
\text { risk }>3.0 \% \\
\text {-Methods: supervised learning; } \\
\text { classification and regression } \\
\text { tree (CART) analysis }\end{array}$ & $\begin{array}{l}\text { Key implications } \\
\text {-Simple CART model with age and bone den- } \\
\text { sity showed similar performance in predict- } \\
\text { ing incident hip fracture compared to the } \\
\text { FRAX risk estimator as the current standard } \\
\text { (AUROC } 0.71 \text { vs. AUROC } 0.70 \text { ). } \\
\text {-Simple classification by age and BMD may } \\
\text { have a similar predictive performance to the } \\
\text { FRAX hip fracture risk category. } \\
\text { Limitations } \\
\text {-Potential of overfitting } \\
\text {-Limited statistical power for comparison of } \\
\text { discrimination statistics due to low incidence } \\
\text { of hip fracture. }\end{array}$ \\
\hline & $\begin{array}{l}\text { Basu et al. (2018) } \\
\text { [19] (Diabetes } \\
\text { and related } \\
\text { disorders) }\end{array}$ & $\begin{array}{l}-10,251 \text { ACCORD trial } \\
\text { participants aged } 40 \text { to } 79 \\
\text { years with type } 2 \text { diabetes, } \\
\text { HbA1c } 7.5 \% \text { or higher, or } \\
\text { cardiovascular diseases or } \\
\text { risk factors, those who ran- } \\
\text { domized to target HbA1c } \\
<6.0 \% \text { (intensive) vs. } \\
7.0 \%-7.9 \% \text { (standard } \\
\text { group) }\end{array}$ & $\begin{array}{l}\text {-Aim: to identify subgroups with } \\
\text { a heterogeneous treatment effect } \\
\text { in response to intensive glycemic } \\
\text { therapy } \\
\text {-Reference label: treatment ef- } \\
\text { fect defined as the absolute dif- } \\
\text { ference in the all-cause mortali- } \\
\text { ty rate between the intensive } \\
\text { and standard therapy groups } \\
\text {-Comparator: not applicable } \\
\text {-Methods: supervised learning; } \\
\text { gradient forest analysis }\end{array}$ & $\begin{array}{l}\text { Key implications } \\
\text {-Compared to } 3.7 \% \text { increased mortality by in- } \\
\text { tensive vs. standard therapy in group } 4 \text {, group } \\
1 \text { showed a } 2.3 \% \text { mortality reduction in the } \\
\text { intensive therapy group ( } 95 \% \mathrm{CI},-0.2 \% \text { to } \\
4.5 \% \text { ), which made the obvious contrast with } \\
\text { the main result from the study. } \\
\text {-Identified characteristics of patients who may } \\
\text { have benefited from intensive glycemic ther- } \\
\text { apy (younger individuals with relatively low } \\
\text { hemoglycosylation index) } \\
\text {-Offered an example to find, clinically mean- } \\
\text { ingful subgroups with heterogeneous treat- } \\
\text { ment effects using data from randomized } \\
\text { trials. } \\
\text { Limitations } \\
\text {-Post hoc analysis of a single trial that was } \\
\text { conducted before the development of recent } \\
\text { diabetes medications with cardiovascular } \\
\text { benefits. }\end{array}$ \\
\hline & $\begin{array}{l}\text { Fan et al. (2019) } \\
\text { [20] (Pituitary } \\
\text { diseases) }\end{array}$ & $\begin{array}{l}\text {-Retrospective cohort of } 668 \\
\text { patients with acromegaly } \\
\text { included age, gender, } \\
\text { hypertension, blood } \\
\text { glucose, laboratory values, } \\
\text { maximal tumor diameter, } \\
\text { bilateral Knosp grade based } \\
\text { on magnetic resonance } \\
\text { imaging findings, and } \\
\text { surgical methods } \\
\text {-Internal validation set } \\
(n=134)\end{array}$ & $\begin{array}{l}\text {-Aim: to develop an ML model } \\
\text { for preoperative prediction of } \\
\text { transsphenoidal surgery } \\
\text { response in patients with } \\
\text { acromegaly } \\
\text {-Reference label: remission } \\
\text { (at } 3 \text { months after surgery, either } \\
\text { nadir growth hormone }<4 \mathrm{ng} / \mathrm{mL} \\
\text { after oral glucose tolerance test } \\
\text { or GH < } 1.0 \mathrm{ng} / \mathrm{mL} \text { in a random } \\
\text { sample with normal IGF-1 } \\
\text { levels) } \\
\text {-Comparator: Knosp grade } \\
\text {-Methods: supervised learning; } \\
\text { random forest, logistic regres- } \\
\text { sion, logistic generalized addi- } \\
\text { tive models, gradient boosting } \\
\text { decision tree, gradient boosting } \\
\text { decision tree, adaptive boosting, } \\
\text { extreme gradient boost model }\end{array}$ & $\begin{array}{l}\text { Key implications } \\
\text {-The ML model predicted remission after } \\
\text { surgery better than standard Knosp grade } \\
\text { (AUROC } 0.82 \text { vs. } 0.71 \text { ). } \\
\text {-Showed an exemplary case of applying } \\
\text { various types of ML algorithms in endocrine } \\
\text { diseases with relatively low frequency. } \\
\text { Limitations } \\
\text {-Single-center study } \\
\text {-Limited by short study follow-up duration } \\
\text { (remission determined at } 3 \text { months) } \\
\text {-Omitted radiomics features }\end{array}$ \\
\hline
\end{tabular}


Table 2. Continued

\begin{tabular}{|c|c|c|c|c|}
\hline Task & Study (disease field) & Study subjects & Design and method & Key finding and limitation \\
\hline & $\begin{array}{c}\text { Zaborek et al. } \\
\text { (2019) [21] } \\
\text { (Thyroid } \\
\text { diseases) }\end{array}$ & $\begin{array}{l}\text {-Retrospective cohort of } 598 \\
\text { patients who underwent to- } \\
\text { tal or completion thyroidec- } \\
\text { tomy with pathology show- } \\
\text { ing benign thyroid disease } \\
\text {-Initiated levothyroxine at } \\
1.6 \mu \mathrm{g} / \mathrm{kg} / \text { day, with subse- } \\
\text { quent dose titration at 6- to } \\
\text { 8-week intervals } \\
\text {-Internal validation with } 10 \text { - } \\
\text { fold cross-validation }\end{array}$ & $\begin{array}{l}\text {-Aim: to develop an ML-based } \\
\text { levothyroxine dosing scheme } \\
\text { after total thyroidectomy to } \\
\text { achieve euthyroidism } \\
\text {-Reference label: electronic } \\
\text { health record-based euthyroid } \\
\text { dosing } \\
\text {-Comparator: standard weight- } \\
\text { based dosing } \\
\text {-Methods: supervised learning; } \\
\text { support vector machine, } \\
\text { Bayesian recurrent neural } \\
\text { network, decision trees, random } \\
\text { forests, ordinary least squares } \\
\text { regression, Poisson regression, } \\
\text { gamma regression, ridge regres- } \\
\text { sion, LASSO }\end{array}$ & $\begin{array}{l}\text { Key implications } \\
\text {-The predictive accuracy of the dose-sugges- } \\
\text { tion algorithm was modest ( } 64.8 \%) \text {, which } \\
\text { was better than standard weight-based dosing } \\
(51.3 \%) \text {. } \\
\text {-Provided an ML algorithm to suggest dosing } \\
\text { scheme of levothyroxine after total thyroid- } \\
\text { ectomy, with better accuracy across body } \\
\text { mass index levels } \\
\text { Limitations } \\
\text {-Limited to dataset from a single institution; } \\
\text { need further validation in an external dataset } \\
\text {-Missing information regarding genetic } \\
\text { factors and drug compliance; may hinder } \\
\text { applicability to the real-world setting. }\end{array}$ \\
\hline & $\begin{array}{l}\text { Oroojeni Moham- } \\
\text { mad Javad et al., } \\
\text { (2019) [5] } \\
\text { (Diabetes and } \\
\text { related disorders) }\end{array}$ & $\begin{array}{l}\text {-Medical records of } 87 \\
\text { patients with type } 1 \text { diabetes } \\
\text { from Mass General Hospi- } \\
\text { tal; data for each patient's } \\
\text { visits over a } 10 \text {-year period } \\
\text { (training set) between } 2003 \\
\text { to } 2013 \text {; HbA1c, body mass } \\
\text { index, activity level, } \\
\text { alcohol usage status, insulin } \\
\text { (Lantus) dose } \\
\text {-External validation with } 60 \\
\text { cases }\end{array}$ & $\begin{array}{l}\text {-Aim: to explore an effective re- } \\
\text { inforcement learning frame- } \\
\text { work for determining the opti- } \\
\text { mal long-acting insulin dose for } \\
\text { patients with type } 1 \text { diabetes } \\
\text {-Reference label: physician-pre- } \\
\text { scribed insulin dose } \\
\text {-Comparator: not applicable } \\
\text {-Methods: reinforcement learn- } \\
\text { ing; Q-learning with reward } \\
\text { function set from HbAlc status } \\
\text { at the visit and change of } \\
\text { HbA1c from the past visit }\end{array}$ & $\begin{array}{l}\text { Key implications } \\
\text {-The physician-prescribed insulin dose was } \\
\text { within the dosing interval recommended by } \\
\text { the Q-learning algorithm in } 88 \% \text { of test } \\
\text { cases. } \\
\text {-A proof-of-concept study to provide clinical } \\
\text { decision support for determining insulin dose } \\
\text { in patients with type } 1 \text { diabetes, by applying } \\
\text { reinforcement learning algorithm } \\
\text { Limitations } \\
\text {-Limited by omitting lifestyle information } \\
\text { regarding diet, stress, and medication } \\
\text { adherence } \\
\text {-A relatively small training set } \\
\text {-Only one type of insulin (Lantus) was } \\
\text { examined in the model. }\end{array}$ \\
\hline $\begin{array}{l}\text { Translational } \\
\text { research }\end{array}$ & $\begin{array}{l}\text { Liu et al. (2020) } \\
\text { [22] (Diabetes } \\
\text { and related } \\
\text { disorders) }\end{array}$ & $\begin{array}{l}\text {-20 Drug-naive individuals } \\
\text { with prediabetes (discovery } \\
\text { cohort) } \\
\text {-Determined exercise } \\
\text { responders and non- } \\
\text { responders after } 12 \text {-week } \\
\text { high-intensity exercise } \\
\text { training } \\
\text {-Collected pre- and post- } \\
\text { exercise period feces to } \\
\text { analyze gut microbiota } \\
\text { profile } \\
\text {-Internal validation with } \\
\text { 10-fold cross-validation }\end{array}$ & $\begin{array}{l}\text {-Aim: to find an ML model for } \\
\text { predicting exercise responsive- } \\
\text { ness determined from exercise- } \\
\text { induced alterations in the gut } \\
\text { microbiota } \\
\text {-Reference label: responders } \\
\text { defined as a decrease in the } \\
\text { homeostatic model assessment } \\
\text { of insulin resistance greater than } \\
\text { two-fold technical error } \\
\text {-Comparator: not applicable } \\
\text {-Methods: supervised learning; } \\
\text { random forest model }\end{array}$ & $\begin{array}{l}\text { Key implications } \\
\text {-The ML model identified } 14 \text { microbiome } \\
\text { species and } 15 \text { metabolites from human feces } \\
\text { were able to predict exercise responsiveness } \\
\text { (AUROC } 0.75 \text { in the validation set). } \\
\text {-Provide an example of applying ML } \\
\text { principles to human-to-mice translational } \\
\text { study based on microbiome dataset } \\
\text { Limitations } \\
\text {-Relatively small sample size } \\
\text {-Limited to Chinese males only } \\
\text {-Need further validation in different } \\
\text { population set }\end{array}$ \\
\hline
\end{tabular}

(Continued to the next page) 
Table 2. Continued

\begin{tabular}{|c|c|c|c|c|}
\hline Task & Study (disease field) & Study subjects & Design and method & Key finding and limitation \\
\hline & $\begin{array}{l}\text { Williams et al. } \\
\text { (2019) [23] } \\
\text { (Miscellaneous) }\end{array}$ & $\begin{array}{l}\text {-Prospectively collected data } \\
\text { from archived samples, } \\
\text { clinical data, with approxi- } \\
\text { mately } 85 \text { million protein } \\
\text { measurements in } 16,894 \\
\text { participants from various } \\
\text { cohorts including UK } \\
\text { Whitehall II, Fenland, } \\
\text { HUNT3, US Covance, } \\
\text { HERITAGE Family studies } \\
\text {-70\% Derivation set (with } \\
\text { five repeats of } 10 \text {-fold } \\
\text { cross-validation), } 15 \% \\
\text { refinement set, and } 15 \% \\
\text { validation set for large } \\
\text { (thousands) cohort } \\
-80 \% \text { Derivation set (with } \\
10-\text { fold cross-validation); } \\
20 \% \text { validation set for } \\
\text { smaller dataset (hundreds) }\end{array}$ & $\begin{array}{l}\text {-Aim: to develop plasma protein- } \\
\text { phenotype models for } 11 \text { differ- } \\
\text { ent health indicators (focusing } \\
\text { on percentage body fat and inci- } \\
\text { dent cardiovascular events as } \\
\text { outcomes) } \\
\text {-Reference label: percentage } \\
\text { body fat measured by DXA; } \\
\text { incident cardiovascular events } \\
\text { ascertained in each cohort } \\
\text {-Comparator: not applicable } \\
\text {-Methods: supervised learning; } \\
\text { dimensionality reduction by } \\
\text { false-recovery rate-corrected } P \\
\text { values, proportional hazards } \\
\text { elastic net models }\end{array}$ & $\begin{array}{l}\text { Key implications } \\
\text {-The ML algorithm found proteins associated } \\
\text { with body fat percentage (leptin, FABP, } \\
\text { SFRP4) and CV events (gelsolin, antithrom- } \\
\text { bin III, sTREM-1). } \\
\text {-Reveals the potential of ML algorithm } \\
\text { application to find novel proteomics-based } \\
\text { biomarkers in large-scale, well-established } \\
\text { cohorts. } \\
\text { Limitations } \\
\text {-Caucasian bias in some cohorts; may not be } \\
\text { generalizable to different populations. } \\
\text {-Need future investigation for examining the } \\
\text { sensitivity of current research findings for } \\
\text { longitudinal changes in health status or risks }\end{array}$ \\
\hline & $\begin{array}{l}\text { Shomorony et al. } \\
\text { (2020) [6] } \\
\text { (Miscellaneous) }\end{array}$ & $\begin{array}{l}\text {-1,385 Data features using a } \\
\text { multimodal dataset collect- } \\
\text { ed from 1,253 individuals } \\
\text { including data of whole- } \\
\text { genome sequencing, micro- } \\
\text { biome sequencing, global } \\
\text { metabolome, insulin resis- } \\
\text { tance, whole body and brain } \\
\text { magnetic resonance imag- } \\
\text { ing, bone densitometry, } \\
\text { computed tomography } \\
\text { scans, routine clinical labo- } \\
\text { ratory tests, family history } \\
\text { of disease and medication, } \\
\text { and anthropometric } \\
\text { measurements } \\
\text {-External validation set: } \\
\text { 1,083 individuals from a } \\
\text { separate cohort (TwinsUK } \\
\text { registry) }\end{array}$ & $\begin{array}{l}\text {-Aim: to identify multimodal } \\
\text { biomarker signatures of health } \\
\text { and disease risk using the unsu- } \\
\text { pervised approach } \\
\text {-Reference label: not applicable } \\
\text {-Comparator: not applicable } \\
\text {-Methods: unsupervised learn- } \\
\text { ing; Louvain community detec- } \\
\text { tion, graphical LASSO for net- } \\
\text { work analysis, Markov network } \\
\text { analysis }\end{array}$ & $\begin{array}{l}\text { Key implications } \\
\text {-1-stearoyl-2-dihomo-linolenoyl-GPC and } \\
\text { 1-(1-enyl-palmitoyl)-2-oleoyl-GPC were } \\
\text { identified as novel biomarkers for diabetes, } \\
\text { whereas cinnamoylglycine showed a novel } \\
\text { association with lean mass percentage. } \\
\text {-Provided an example of applying unsuper- } \\
\text { vised learning algorithms to find novel } \\
\text { associations and biomarker signatures associ- } \\
\text { ated with health and disease statues in a large, } \\
\text { multimodal dataset } \\
\text { Limitations } \\
\text {-Underpowered to detect the effects of poly- } \\
\text { genic risk scores based on common variants } \\
\text { for certain traits (explaining a relatively small } \\
\text { fraction of the phenotypic variance) }\end{array}$ \\
\hline
\end{tabular}

ML, machine learning; AUROC, area under the receiver operating characteristic curve; CT, computed tomography; BMD, bone mineral density; DXA, dual-energy X-ray absorptiometry; IGF-1, insulin-like growth factor-1; NAFLD, nonalcoholic fatty liver disease; NASH, nonalcoholic steatohepatitis; LC-MS/MS, liquid chromatography-mass spectrometry; ACCORD, Action to Control Cardiovascular Risk in Diabetes; ALLHAT, Antihypertensive and Lipid-Lowering Treatment to Prevent Heart Attack Trial; WATCH-DM, Weight, Age, hyperTension, Creatinine, High-density lipoprotein cholesterol, Diabetes control, and Myocardial infarction; MrOS, The Osteoporotic Fractures in Men; FRAX, Fracture Risk Assessment Tool; CI, confidence interval; GH, growth hormone; LASSO, least absolute shrinkage and selection operator; HUNT3, the third Nord-Trøndelag Health Study; HERITAGE, HEalth, RIsk factors, exercise Training And GEnetics; FABP, fatty-acid-binding proteins; SFRP4, Secreted frizzled-related protein 4; CV, cardiovascular; sTREM-1, soluble triggering receptor expressed on myeloid cells-1; GPC, glycerophosphocholine. 


\section{Finding novel disease clusters and associations}

Although unsupervised learning has been utilized less often than supervised learning for diagnosis and screening, it may be helpful to find novel clusters and associations within a given dataset. Kruse et al. [16] applied unsupervised hierarchical agglomerative clustering to find groups with high and low risks of fracture in women from a national Danish patient database based on BMD, medication reimbursement, anthropometric characteristics, and comorbidities. Among the nine clusters that were identified, four clusters classified as corresponding to a high risk of fracture showed heterogeneous compliance to antiresorptive treatments, even with a similar distribution of BMD. The age of 60 years was the earliest time point that allowed a clear discrimination between high and average fracture risk. Altogether, that study provided novel insights regarding characteristics related to compliance with bone medications and the optimal age to recommend dual-energy X-ray absorptiometry screening.

\section{Risk prediction}

\section{Clinical outcomes}

Accurately predicting clinical outcomes enables an individualized approach to treatment strategy and monitoring. The Weight, Age, hyperTension, Creatinine, High-density lipoprotein cholesterol, Diabetes control, and Myocardial infarction (WATCHDM) score was developed to predict heart failure risk among patients with type 2 diabetes using ML algorithms based on the Action to Control Cardiovascular Risk in Diabetes (ACCORD) trial dataset, and showed good predictive performance with an external validation set (the Antihypertensive and Lipid-Lowering Treatment to Prevent Heart Attack Trial [ALLHAT]) [17]. Su et al. [18] found that a simple model including only age and BMD selected by classification and regression tree analysis performed similarly to Fracture Risk Assessment Tool (FRAX) categories as a reference tool for predicting incident hip fracture in a large cohort of community-dwelling older men.

\section{Treatment responses}

ML principles can be used to find specific subgroups with a heterogeneous response to treatment. Basu et al. [19] re-analyzed the ACCORD trial data to find subgroups with different treatment effects in response to intensive glucose control compared to standard therapy. Although intensive glucose control was associated with increased mortality in the ACCORD trial published in 2008, their post hoc analysis identified that a subgroup of patients experienced a survival benefit from intensive treatment, and the proportion of patients in the subgroup that made the main contribution to increased mortality in the trial was relatively small. This study provides an example of the utility of ML in dissecting treatment responses, with the potential for a more tailored approach both for interpreting results from previous trials and for applying therapeutic strategies according to individual status. For the prediction of treatment response in patients with acromegaly, anthropometric and biochemical data with imaging features were combined in an ML model that achieved better prognostication than the reference prediction tool [20]. Good ML models may have the potential to provide guidance for dose adjustment, particularly in patients with chronic conditions requiring the indefinite replacement of certain hormones, as in patients who receive thyroid hormone replacement after total thyroidectomy or in type 1 diabetes patients who receive insulin replacement. Zaborek et al. [21] built a supervised ML model to guide levothyroxine dose adjustment, which showed a fair improvement of predictive accuracy compared to the current standard of weight-based dosing. A reinforcement training algorithm has been applied to guide the optimal dosing of long-acting insulin in patients with type 1 diabetes [5]. Although the results are preliminary, these studies illustrate the ongoing efforts made by endocrinology researchers to improve patient care by achieving better predictions of the disease course and response to treatment.

\section{Translational research}

ML algorithms have become a crucial methodology in translational research with the rise of the multi-omics approach, which produces abundant datasets with numerous features to be accounted for. Liu et al. [22] used an ML algorithm to find key microbiota species and metabolites highly related to exercise responsiveness in humans. Human exercise responders and nonresponders had different patterns of exercise-induced alterations in the gut microbiota, and fecal microbial transplantation from responders to mice conferred the benefits of exercise on insulin sensitivity. A random forest algorithm was used to select 19 features (14 species and 15 metabolites) showing a major difference between the exercise-responsive and nonresponsive groups among thousands of microbiota species and metabolites, and these features have the potential to be utilized as biomarkers for personalized responses to exercise. Another study aimed to discover proteomics-based biomarkers for 11 health outcomes, including percentage body fat, lean mass, current smoking, and risk of incident cardiovascular outcomes [23]. By combining large, well-established, community-based cohort databases and samples, the authors took a comprehensive approach to find 
highly predictive proteins and related models using elegant MLbased techniques, although the actual applicability of these findings needs to be validated in long-term studies in different populations. Unsupervised learning was also applied to find significant associations and interactions among multimodal datasets, providing novel insights for potential metabolite biomarkers of diabetes and sarcopenia [6].

\section{CONCLUSIONS}

High-quality ML-based endocrinology research, like research in other medical fields, requires a clear, testable hypothesis based on unmet clinical needs, combined with access to a dataset that provides sufficient information to solve the problem. As Kim et al. [30] clearly addressed in a previous issue of this journal, the ability to access a large volume of medical data itself does not necessarily enable (or mandate) an ML-based approach due to the inherently unrefined, heterogeneous nature of most current medical datasets. Well-designed, timely study designs based on clinical expertise, an emphasis on using a standardized approach to control data quality (beyond a focus on data quantity alone and methodological complexity), collaboration and open communication between clinical domain experts and ML engineers, developing interpretable ML models in contrast to the black-box nature of some algorithms, and creating a supportive environment with input from government, profit or non-profit sectors, study participants, and patients are the core prerequisites for the promising changes that are expected in clinical practice in the field of endocrinology and metabolism through the convergence of artificial and human intelligence [31]. The role of endocrinologists as domain experts will remain crucial for achieving these prerequisites by examining the true clinical impact of flourishing ML-based research products in prospective studies and by ensuring the scientific rigor needed to the benefits of this convergence for patients who suffer from endocrine diseases.

\section{CONFLICTS OF INTEREST}

No potential conflict of interest relevant to this article was reported.

\section{ACKNOWLEDGMENTS}

This work was supported by a grant of the Korea Health Technology R\&D Project through the Korea Health Industry Development Institute (KHIDI), funded by the Ministry of Health \&
Welfare, Republic of Korea (grant number: HI18C0474). We thank Minheui Yu and Doori Cho, the members of the SENTINEL (Severance ENdocrinology daTa scIeNcE pLatform) team for technical assistance in searching and summarizing the relevant literature (4-2018-1215).

\section{ORCID}

Namki Hong https://orcid.org/0000-0002-8246-1956

\section{REFERENCES}

1. McCarthy J. From here to human-level AI. Artif Intell 2007; 171:1174-82.

2. McCarthy J. What is artificial intelligence? [Internet]. Stanford: Stanford University; 2007 [cited 2020 Feb 24]. Available from: http://www-formal.stanford.edu/jmc/whatisai/.

3. Beaulieu-Jones B, Finlayson SG, Chivers C, Chen I, McDermott M, Kandola J, et al. Trends and focus of machine learning applications for health research. JAMA Netw Open 2019;2:e1914051.

4. Artzi NS, Shilo S, Hadar E, Rossman H, Barbash-Hazan S, Ben-Haroush A, et al. Prediction of gestational diabetes based on nationwide electronic health records. Nat Med 2020;26:71-6.

5. Oroojeni Mohammad Javad M, Agboola SO, Jethwani K, Zeid A, Kamarthi S. A reinforcement learning-based method for management of type 1 diabetes: exploratory study. JMIR Diabetes 2019;4:e12905.

6. Shomorony I, Cirulli ET, Huang L, Napier LA, Heister RR, Hicks $\mathrm{M}$, et al. An unsupervised learning approach to identify novel signatures of health and disease from multimodal data. Genome Med 2020;12:7.

7. Ballinger B, Hsieh J, Singh A, Sohoni N, Wang J, Tison GH, et al. DeepHeart: semi-supervised sequence learning for cardiovascular risk prediction [Internet]. arXiv; 2018 [cited 2020 Feb 24]. Available from: https://arxiv.org/abs/1802.02511.

8. Dinga R, Penninx BW, Veltman DJ, Schmaal L, Marquand AF. Beyond accuracy: measures for assessing machine learning models, pitfalls and guidelines. bioRxiv 2019:743138. https://doi.org/10.1101/743138.

9. Handelman GS, Kok HK, Chandra RV, Razavi AH, Huang S, Brooks M, et al. Peering into the black box of artificial intelligence: evaluation metrics of machine learning methods. AJR Am J Roentgenol 2019;212:38-43.

10. Saito T, Rehmsmeier M. The precision-recall plot is more in- 
formative than the ROC plot when evaluating binary classifiers on imbalanced datasets. PLoS One 2015;10:e0118432.

11. Chicco D, Jurman G. The advantages of the Matthews correlation coefficient (MCC) over F1 score and accuracy in binary classification evaluation. BMC Genomics 2020;21:6.

12. Valentinitsch A, Trebeschi S, Kaesmacher J, Lorenz C, Loffler MT, Zimmer C, et al. Opportunistic osteoporosis screening in multi-detector CT images via local classification of textures. Osteoporos Int 2019;30:1275-85.

13. Kong X, Gong S, Su L, Howard N, Kong Y. Automatic detection of acromegaly from facial photographs using machine learning methods. EBioMedicine 2018;27:94-102.

14. Buda M, Wildman-Tobriner B, Hoang JK, Thayer D, Tessler FN, Middleton WD, et al. Management of thyroid nodules seen on US images: deep learning may match performance of radiologists. Radiology 2019;292:695-701.

15. Perakakis N, Polyzos SA, Yazdani A, Sala-Vila A, Kountouras J, Anastasilakis AD, et al. Non-invasive diagnosis of nonalcoholic steatohepatitis and fibrosis with the use of omics and supervised learning: a proof of concept study. Metabo$\operatorname{lism} 2019 ; 101: 154005$.

16. Kruse C, Eiken P, Vestergaard P. Clinical fracture risk evaluated by hierarchical agglomerative clustering. Osteoporos Int 2017;28:819-32.

17. Segar MW, Vaduganathan M, Patel KV, McGuire DK, Butler $\mathrm{J}$, Fonarow GC, et al. Machine learning to predict the risk of incident heart failure hospitalization among patients with diabetes: the WATCH-DM risk score. Diabetes Care 2019;42: 2298-306.

18. Su Y, Kwok TC, Cummings SR, Yip BH, Cawthon PM. Can classification and regression tree analysis help identify clinically meaningful risk groups for hip fracture prediction in older American men (the MrOS cohort study)? JBMR Plus 2019;3:e10207.

19. Basu S, Raghavan S, Wexler DJ, Berkowitz SA. Characteristics associated with decreased or increased mortality risk from glycemic therapy among patients with type 2 diabetes and high cardiovascular risk: machine learning analysis of the ACCORD trial. Diabetes Care 2018;41:604-12.

20. Fan Y, Li Y, Li Y, Feng S, Bao X, Feng M, et al. Development and assessment of machine learning algorithms for predicting remission after transsphenoidal surgery among patients with acromegaly. Endocrine 2020;67:412-22.

21. Zaborek NA, Cheng A, Imbus JR, Long KL, Pitt SC, Sippel $\mathrm{RS}$, et al. The optimal dosing scheme for levothyroxine after thyroidectomy: a comprehensive comparison and evaluation. Surgery 2019;165:92-8.

22. Liu Y, Wang Y, Ni Y, Cheung CK, Lam KS, Wang Y, et al. Gut microbiome fermentation determines the efficacy of exercise for diabetes prevention. Cell Metab 2020;31:77-91.

23. Williams SA, Kivimaki M, Langenberg C, Hingorani AD, Casas JP, Bouchard C, et al. Plasma protein patterns as comprehensive indicators of health. Nat Med 2019;25:1851-7.

24. De Silva K, Jonsson D, Demmer RT. A combined strategy of feature selection and machine learning to identify predictors of prediabetes. J Am Med Inform Assoc 2020;27:396406.

25. Somnay YR, Craven M, McCoy KL, Carty SE, Wang TS, Greenberg CC, et al. Improving diagnostic recognition of primary hyperparathyroidism with machine learning. Surgery 2017;161:1113-21.

26. Gulshan V, Peng L, Coram M, Stumpe MC, Wu D, Narayanaswamy A, et al. Development and validation of a deep learning algorithm for detection of diabetic retinopathy in retinal fundus photographs. JAMA 2016;316:2402-10.

27. Eastell R, Brandi ML, Costa AG, D’Amour P, Shoback DM, Thakker RV. Diagnosis of asymptomatic primary hyperparathyroidism: proceedings of the Fourth International Workshop. J Clin Endocrinol Metab 2014;99:3570-9.

28. Akkus Z, Cai J, Boonrod A, Zeinoddini A, Weston AD, Philbrick KA, et al. A survey of deep-learning applications in ultrasound: artificial intelligence-powered ultrasound for improving clinical workflow. J Am Coll Radiol 2019;16(9 Pt B):1318-28.

29. Katsiki N, Gastaldelli A, Mikhailidis DP. Predictive models with the use of omics and supervised machine learning to diagnose non-alcoholic fatty liver disease: a "non-invasive alternative" to liver biopsy? Metabolism 2019;101:154010.

30. Kim HS, Kim DJ, Yoon KH. Medical big data is not yet available: why we need realism rather than exaggeration. Endocrinol Metab (Seoul) 2019;34:349-54.

31. Topol EJ. High-performance medicine: the convergence of human and artificial intelligence. Nat Med 2019;25:44-56. 